\title{
ARTICLE
}

\section{IL-22-binding protein exacerbates influenza, bacterial super-infection}

Robert N. Abood ${ }^{1}$, Kevin J. McHugh', Helen E. Rich ${ }^{1}$, Marianna A. Ortiz ${ }^{1}$, Joshua M. Tobin ${ }^{1}$, Krishnaveni Ramanan ${ }^{1}$, Keven M. Robinson ${ }^{2}$, Jennifer M. Bomberger ${ }^{3}$, Jay K. Kolls ${ }^{4}$, Michelle L. Manni ${ }^{1}$, Derek A. Pociask ${ }^{5}$ and John F. Alcorn ${ }^{1}$

Secondary bacterial pneumonia is a significant complication of severe influenza infection and Staphylococcus aureus and Streptococcus pneumoniae are the primary pathogens of interest. IL-22 promotes S. aureus and S. pneumoniae host defense in the lung through epithelial integrity and induction of antimicrobial peptides and is inhibited by the soluble decoy receptor IL-22binding protein (IL-22BP). Little is known about the effect of the IL-22/IL-22BP regulatory pathway on lung infection, and it has not been studied in the setting of super-infection. We exposed wild-type and IL-22BP ${ }^{-1-}$ mice to influenza A/PR/8/34 for 6 days prior to infection with S. aureus (USA300) S. pneumoniae. Super-infected IL-22BP ${ }^{-1-}$ mice had decreased bacterial burden and improved survival compared to controls. IL-22BP ${ }^{-1-}$ mice exhibited decreased inflammation, increased lipocalin 2 expression, and deletion of IL-22BP was associated with preserved epithelial barrier function with evidence of improved tight junction stability. Human bronchial epithelial cells treated with IL-22Fc showed evidence of improved tight junctions compared to untreated cells. This study revealed that IL-22 $\mathrm{BP}^{-1-}$ mice are protected during influenza, bacterial super-infection, suggesting that IL-22BP has a proinflammatory role and impairs epithelial barrier function likely through interaction with IL-22

Mucosal Immunology (2019) 12:1231-1243; https://doi.org/10.1038/s41385-019-0188-7

\section{INTRODUCTION}

Lower respiratory infections are the most common infectious cause of death in children and adults worldwide. Many types of pathogens cause respiratory infection, but viruses including influenza are especially prominent. Influenza causes an acute respiratory illness that occurs primarily in the setting of seasonal outbreaks, but it can also occur as pandemics when new strains of virus are introduced to the population. Infection generally causes mild to moderate selflimited disease in otherwise healthy individuals, but severe disease is associated with significant morbidity and mortality. There are 3-5 million cases of severe influenza and 250,000-500,000 influenzarelated deaths worldwide each year, with $12,000-56,000$ deaths in the United States alone'.

Secondary bacterial pneumonia is a widely known complication of severe influenza infection. It has been described as a significant cause of excess mortality during influenza pandemics including those in 1918 and $2009^{2-4}$, and it has been linked to an increase in seasonal influenza-related deaths in children ${ }^{5}$. While Streptococcus pneumonia has historically been the most commonly implicated organism in these super-infections, Staphylococcus aureus has recently emerged as the leading associated pathogen. This appears to be a result of the increasing prevalence of methicillin-resistant S. aureus (MRSA) in the community ${ }^{5-7}$. Community-acquired strains of MRSA including the USA300 clonotype have been shown to be associated with increased mortality in young, healthy individuals with influenza ${ }^{8,9}$.
Interleukin-22 (IL-22) is a cytokine in the IL-10 family that is expressed by cells of the innate and adaptive immune system including $T_{H} 17$ cells. It binds to a heterodimeric receptor (IL-22R) consisting of IL-22Ra1 and IL-10R2, and signaling occurs through phosphorylation of Jak1 and Tyk2 kinases and activation of STAT1 and STAT3 transcription factors ${ }^{10}$. The IL-22 receptor is present on the epithelial surfaces of the lungs, which is a significant source of potential infection due to continuous exposure to the external environment ${ }^{11,12}$. IL-22 promotes immunity in the lungs through epithelial protective effects that limit bacterial spread. IL-22 functions in combination with the Type 17 cytokine, IL-17 to induce antimicrobial peptide (AMP) production and the release of chemokines for phagocyte recruitment. In this way, it has been shown to have a role in host defense against the bacterial pathogens Klebsiella pneumoniae and Streptococcus pneumoniae ${ }^{13,14}$.

A soluble binding protein known as IL-22BP (also IL-22Ra2) competitively inhibits the activity of $\mathrm{IL}-22^{15-17}$. Single-nucleotide polymorphisms in the IL-22BP gene have been associated with multiple sclerosis (MS) and Schistosoma liver infection in humans $^{18,19}$. It has also been shown to impact immunity and microbiome homeostasis within the gastrointestinal tract ${ }^{20}$. The role of IL-22BP in host defense of the lung is less clear. A recent study found that mice treated with an IL-22BP neutralizing antibody had reduced susceptibility to infection and lung damage in a Pseudomonas aeruginosa pneumonia model ${ }^{21}$. To our knowledge, the role of IL-22BP on super-infection in the lung has not been reported.

\footnotetext{
${ }^{1}$ Department of Pediatrics, UPMC Children's Hospital of Pittsburgh, Pittsburgh, PA, USA; ${ }^{2}$ Department of Medicine, University of Pittsburgh Medical Center, Pittsburgh, PA, USA; ${ }^{3}$ Department of Microbiology and Molecular Genetics, University of Pittsburgh, Pittsburgh, PA, USA; ${ }^{4}$ Center for Translational Research in Infection and Inflammation, Tulane

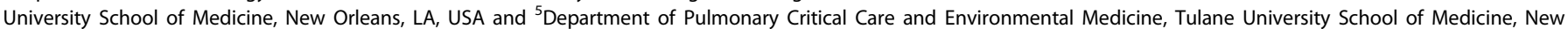
Orleans, LA, USA

Correspondence: John F. Alcorn (john.alcorn@chp.edu)
}

Received: 8 June 2018 Revised: 15 June 2019 Accepted: 24 June 2019

Published online: 11 July 2019 


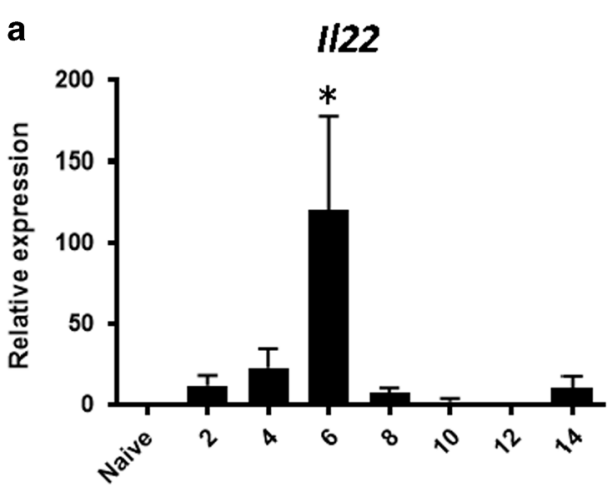

Days post-infection

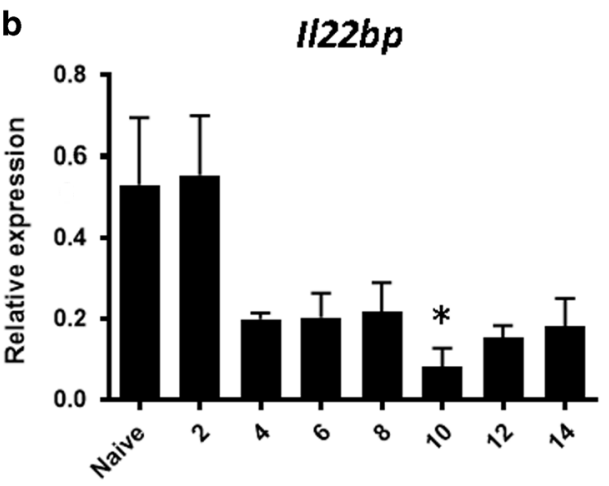

Days post-infection

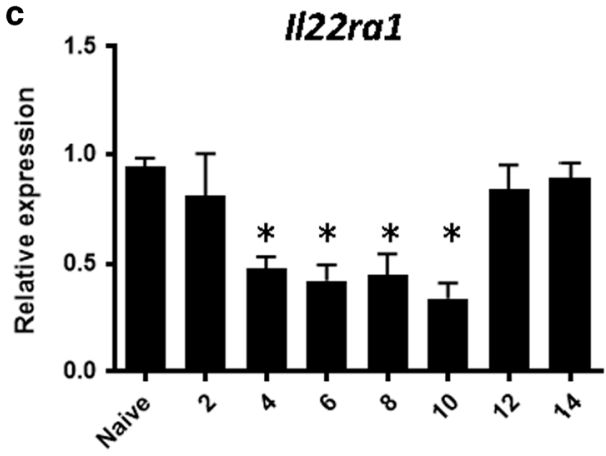

Days post-infection

Fig. 1 The impact of influenza infection on the IL-22 pathway. C57BL/6 mice were infected with $100 \mathrm{PFU}$ of influenza A/PR/8/34 or PBS for 14 days and lungs were harvested every 2 days post-infection. a $1 / 22$ gene expression in lung tissue ( $n=4 /$ time point). b $/ 122 b p$ gene expression in lung tissue ( $n=4 /$ time point). c $1 / 22$ ral gene expression in lung tissue $\left(n=4 /\right.$ time point). ${ }^{*} p<0.05$ Significance was tested by one-way ANOVA

Our group has previously shown that $\mathrm{IL}-22^{-1-}$ mice have impaired bacterial clearance during $S$. aureus pneumonia compared to wild-type mice ${ }^{22}$. During super-infection with influenza $A$ and S. aureus, mice demonstrated reduced production of Type 17 cytokines, including IL-22, in addition to reduced clearance of virus and bacteria $^{22}$. Similarly, it has been established that IL-22 is protective against secondary bacterial infection by S. pneumoniae following influenza A infection ${ }^{23,24}$. However, the specific role of IL-22BP during super-infection of the lung is not known and that is the focus of our current work.

\section{RESULTS}

The kinetics of II22, II22ra1, and II22bp gene expression during influenza infection

In order to determine the role of IL-22BP during influenza and super-infection we interrogated the temporal expression of 1122 and its receptors during influenza infection. The super-infection model utilized is initiated on day 6 post-influenza infection. I/22 expression was significantly increased by influenza infection on day 6 (Fig. 1a). Influenza infection significantly decreased expression of IL-22's signaling (II22ra1) and decoy (II22bp) receptor (Fig. 1b, c), although expression of both receptors was readily detectable at all time points. These data suggest that day 6 postinfluenza is a likely time point to observe maximal effects of IL-22 and/or IL-22BP in our model.

$\mathrm{IL}-22 \mathrm{BP}^{-/-}$mice are protected from influenza infection exacerbated by $S$. aureus or S. pneumoniae

Based on the known protective effects of IL-22, we hypothesized that IL-22BP would have a negative effect on super-infection due to inhibition of IL-22 activity. We tested this hypothesis by infecting wild-type $\mathrm{C} 57 \mathrm{BL} / 6$ and $\mathrm{IL}-22 \mathrm{BP}^{-1-}$ mice with influenza $\mathrm{A} /$ $\mathrm{PR} / 8 / 34$ or vehicle for 6 days prior to infection with S. aureus or vehicle for an additional $24 \mathrm{~h}$. Compared to WT mice, IL-22BP ${ }^{-1-}$ mice were protected against $S$. aureus infection as demonstrated by decreased bacterial burden in the lung (Fig. 2a).

Given the decreased bacterial burden, we set out to determine whether this phenotype provided a morbidity or survival benefit over WT mice. IL-22BP ${ }^{-1-}$ mice did not display altered weight loss to single influenza infection at days 6 or 7 post-infection or superinfection at day 7 post-infection compared to WT mice (Fig. 2b). Following a lethal challenge of influenza and S. aureus, IL-22BP ${ }^{-1-}$ mice were however protected from super-infection-induced mortality compared to WT mice although not completely rescued (Fig. 2c). This result indicates that the observed phenotype of the $\mathrm{IL}-22 \mathrm{BP}^{-/-}$mice is relevant to disease course and outcomes during super-infection.

IL-22 has also been shown to be protective against $S$. pneumoniae during influenza infection ${ }^{23,24}$. We looked to see if we could identify an IL-22BP phenotype in S. pneumoniae secondary bacterial pneumonia that was similar to what was observed with influenza and S. aureus. We infected WT and IL$22 \mathrm{BP}^{-1-}$ mice with influenza for 6 days prior to infection with $S$. pneumoniae for an additional $48 \mathrm{~h}$. IL-22BP ${ }^{-1-}$ mice had decreased bacterial burden compared to WT mice (Fig. 2d). These findings are similar to what was seen in our model of influenza, S. aureus super-infection, but the differences between the two groups were quantitatively more substantial. Most notably, after $48 \mathrm{~h}$ there was nearly complete clearance of $S$. pneumoniae in most IL-22BP ${ }^{-1-}$ mice while the bacterial burden in WT mice remained high. We next examined if bacterial dissemination from the lung differed in 
a

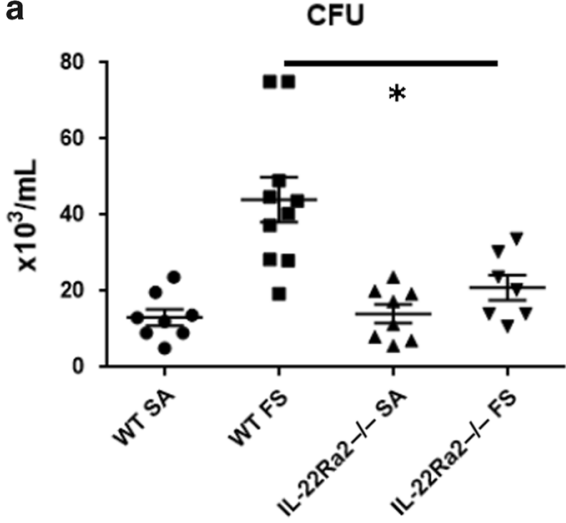

C

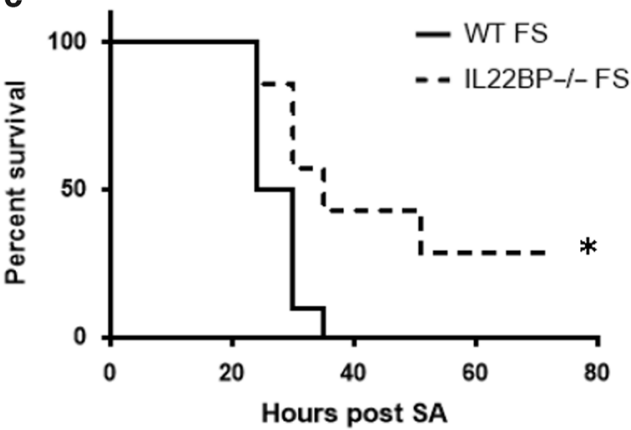

e

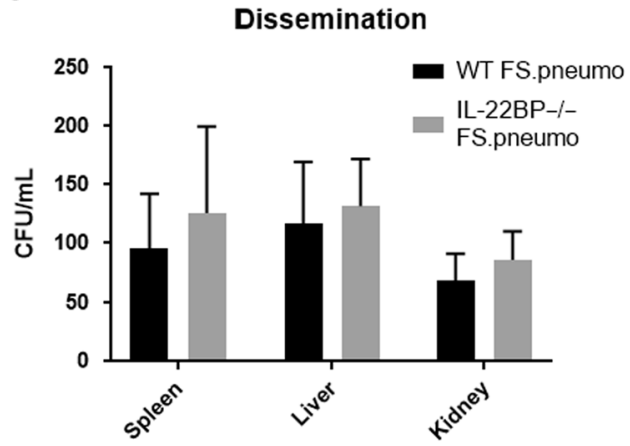

b

Weight loss

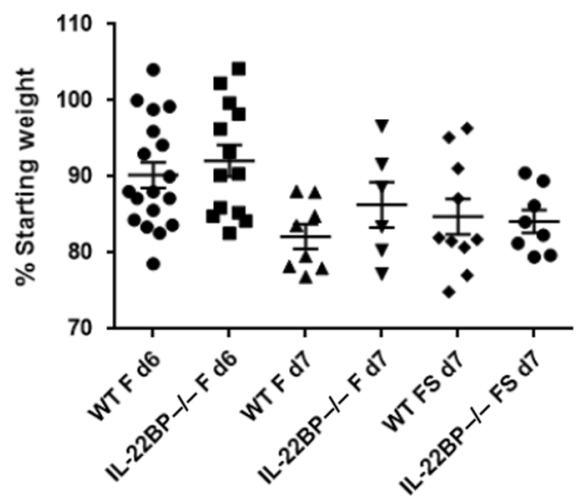

d

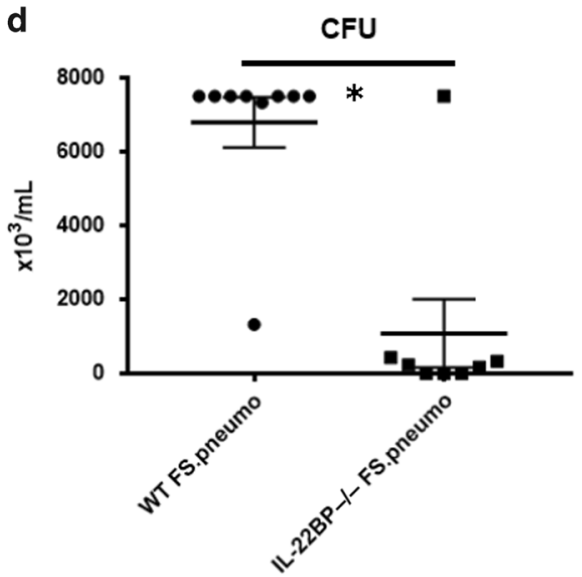

f

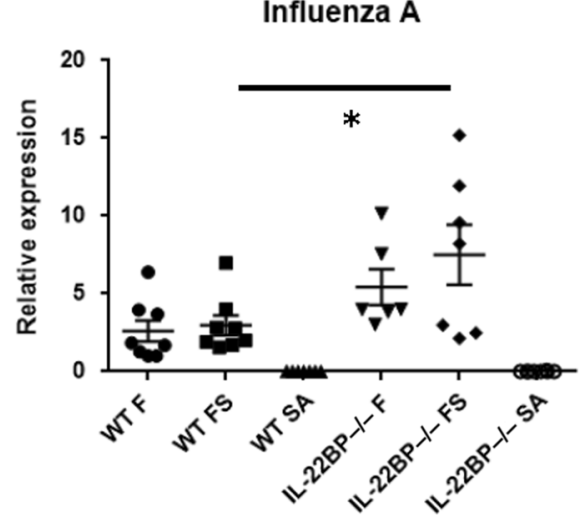

Fig. 2 IL-22BP ${ }^{-1-}$ mice have improved bacterial clearance and decreased mortality. C57BL/6 and IL-22BP ${ }^{-1-}$ mice were infected with $100 \mathrm{PFU}$ of influenza A/PR/8/34 or vehicle for 6 days prior to infection with $5 \times 10^{7} \mathrm{CFU}$ MRSA for $24 \mathrm{~h}$ or $10^{4} \mathrm{CFU} / \mathrm{mL}$ S. pneumoniae for $48 \mathrm{~h}$. SA $=S$. aureus, FS = influenza, S. aureus, F= influenza, FS.pneumo=influenza, S. pneumoniae infection throughout the figures. a MRSA bacterial colony counts in the lung $(n=7-10)$. b Weight loss observed during influenza or super-infection $(n=6-18)$. c Influenza, MRSA survival curve $(n=7-10)$. d S. pneumoniae bacterial colony counts in the lung $(n=8-9)$. e Bacterial dissemination to other organs $(n=3-4)$. $\mathbf{f}$ Influenza matrix protein gene expression measured by RT-PCR $(n=6-8)$. ${ }^{*} p<0.05$ Significance was tested by unpaired $t$-test or one-way ANOVA or Gehan-Brislow-Wilcoxon test. Each experiment was independently performed twice and the data shown are from combined experiments with the exception of the survival curve in panel $\mathbf{c}$ and dissemination in panel $\mathbf{e}$ that are single representative studies

WT and IL-22BP ${ }^{-1-}$ mice. We observed little dissemination to other organs in the influenza, S. aureus model (data not shown); however a greater amount was observed in the pneumococcal model. There were no differences in bacterial dissemination between WT and IL-22 $\mathrm{BP}^{-1-}$ mice (Fig. 2e).

Influenza matrix protein gene expression was then measured as an indicator of viral burden in IL-22BP ${ }^{-1-}$ and WT mice. We found that there was no reduction in influenza viral burden in IL-22 $\mathrm{BP}^{-/-}$ mice, but rather, viral burden was increased compared to WT mice in super-infection (Fig. 2f). These findings suggest that the effect of IL-22BP is on super-infection rather than influenza infection alone.

IL-22BP ${ }^{-1-}$ mice have reduced airway inflammation during superinfection

We next evaluated whether IL-22BP ${ }^{-1-}$ mice demonstrated suppression of inflammation during super-infection by either $S$. aureus or S. pneumoniae. Analysis revealed that IL-22BP ${ }^{-1-}$ mice 

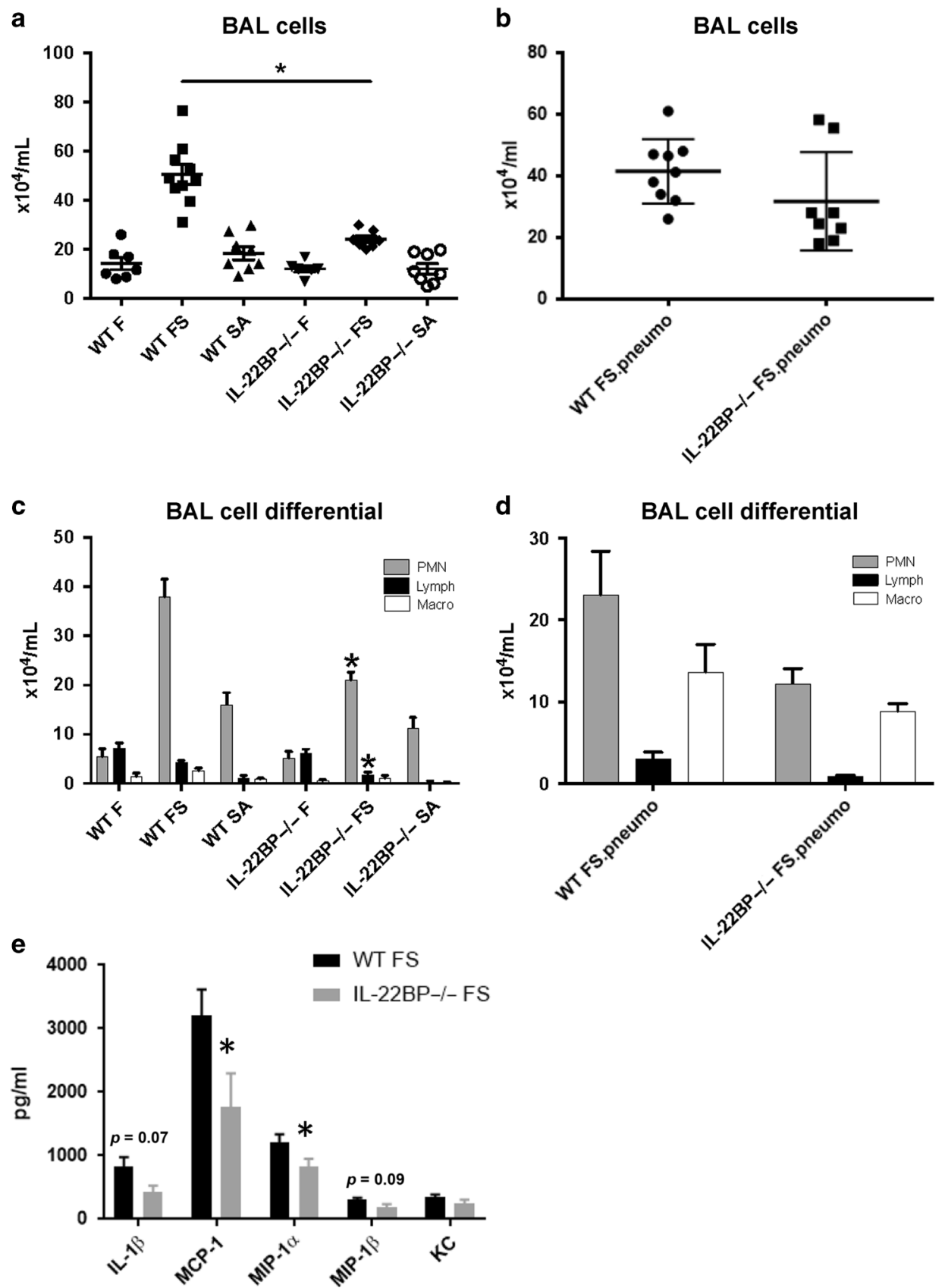

Fig. 3 IL-22BP deficiency decreases inflammation. C57BL/6 and IL-22BP ${ }^{-1-}$ mice were infected with $100 \mathrm{PFU}$ of influenza A/PR/8/34 or vehicle for 6 days prior to infection with $5 \times 10^{7} \mathrm{CFU}$ MRSA for $24 \mathrm{~h}$ or $10^{4} \mathrm{CFU} / \mathrm{mL}$ S. pneumoniae for $48 \mathrm{~h}$. $\mathbf{a}$, b Total cell count in BAL fluid $(n=6-10)$. c, d Differential cell counts in BAL fluid $(n=6-10)$. e Protein production of pro-inflammatory cytokines in lung homogenate measured by Lincoplex $(n=4-6)$. ${ }^{*} p<0.05$ Significance was tested by unpaired $t$-test or one-way ANOVA. Each experiment was independently performed twice and the data shown are from combined experiments

super-infected with influenza and S. aureus had decreased cellular infiltration into the BAL fluid compared to WT mice (Fig. 3a). A trend towards decreased cellular infiltration into the BAL fluid was also noted in IL-22BP ${ }^{-1-}$ mice super-infected with influenza and $S$. pneumoniae (Fig. 3b). Differential cell counts from the BAL fluid showed reduced neutrophil and lymphocyte accumulation in the lung of IL-22 $\mathrm{BP}^{-/-}$mice compared to WT mice. There was a trend towards reduced accumulation of macrophages in $\mathrm{IL}-22 \mathrm{BP}^{-1-}$ mice that did not reach statistical significance (Fig. $3 c$ ). In the influenza, S. pneumoniae model, differential cell counts showed a trend towards reduced neutrophil accumulation in the IL-22BP ${ }^{-1-}$ mice that were not statistically significant (Fig. 3d). The decreased cellular infiltration in IL-22 $\mathrm{BP}^{-1-}$ mice was also associated with a reduction in pro-inflammatory cytokines. Quantities of MCP-1, a regulator of the monocyte inflammatory response, were reduced in IL-22BP ${ }^{-1-}$ mice; as were quantities of MIP-1a, a mediator of neutrophil activation and recruitment (Fig. 3e). Protein levels of IL$1 \beta$ and MIP-1 $\beta$ trended towards reduction in $\mathrm{IL}-22 \mathrm{BP}^{-1-}$ mice, while the neutrophil chemoattractant $\mathrm{KC}$ was unchanged. These data provide evidence for reduced airway inflammation in superinfected IL-22BP ${ }^{-1-}$ mice with a specific reduction in neutrophil recruitment and accumulation. 

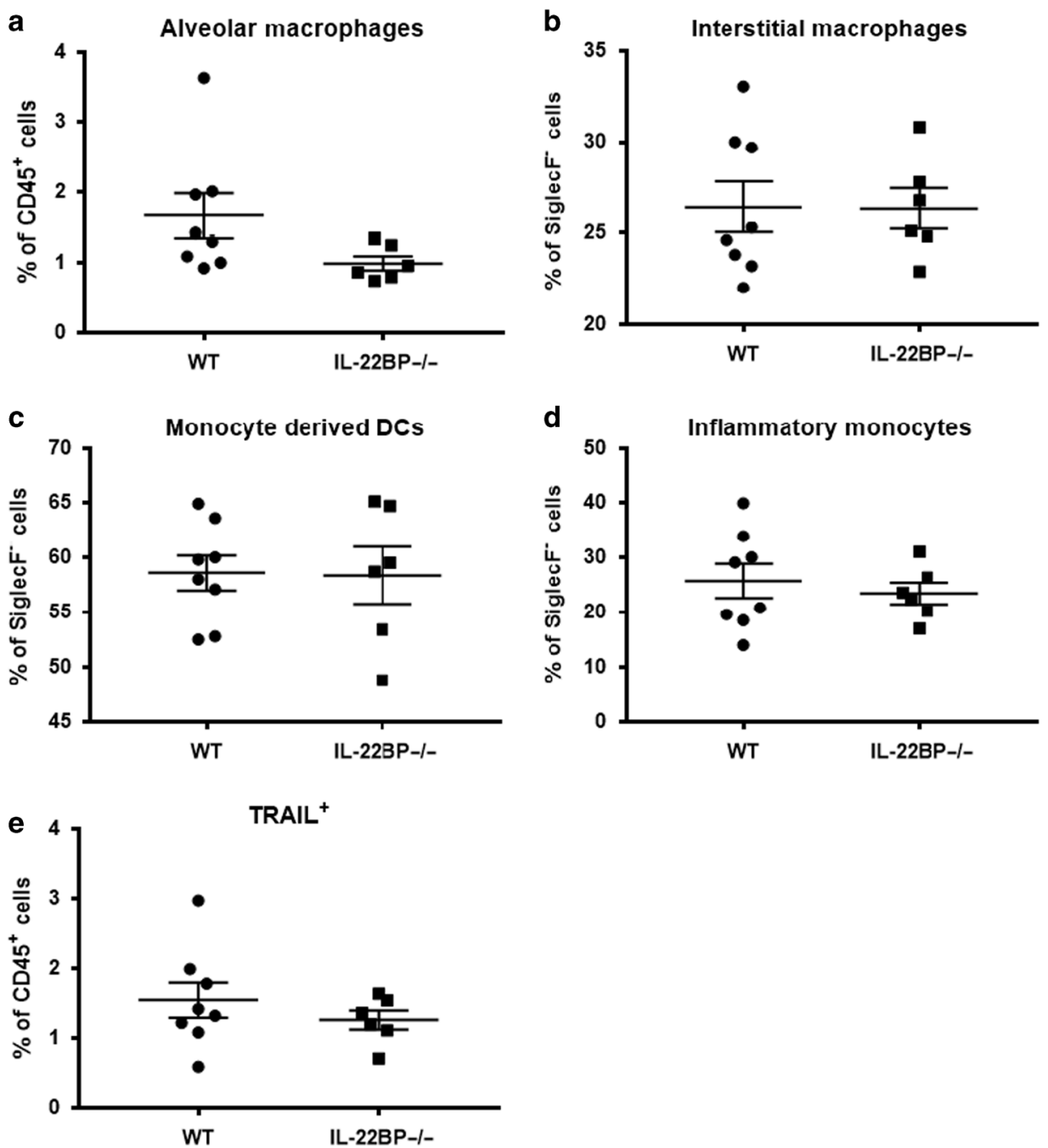

Fig. 4 Deletion of IL-22BP did not impact monocyte subpopulations in the lung. C57BL/ 6 and IL-22BP ${ }^{-1-}$ mice were infected with 100 PFU of influenza $A / P R / 8 / 34$ or vehicle for 6 days prior to infection with $5 \times 10^{7}$ CFU MRSA for $24 \mathrm{~h}$. Mouse lungs were homogenized to single cell suspensions and stained for analysis of monocyte populations by flow cytometry. a Alveolar macrophages. b Interstitial macrophages. c Inflammatory monocytes. d Monocyte-derived dendritic cells. e TRAIL-positive monocytes $(n=6-8)$. Data are shown from a single representative study

Deletion of IL-22BP did not impact the frequency of monocytes in the lung during super-infection

In order to potentially explain the improved bacterial clearance in WT IL-22BP ${ }^{-1-}$ compared to WT mice, we examined if the cellular identities of the monocytes in the lung were altered. Different classes of inflammatory monocytes have been shown to drive lung injury and epithelial apoptosis and leak ${ }^{25-28}$. We performed flow cytometry analysis to examine the frequency of alveolar macrophages, inflammatory monocytes, interstitial macrophages, and monocyte-derived dendritic cells in our model. Somewhat surprisingly, there were no differences in these cell populations in the lung (Fig. 4). There were few alveolar macrophages present at this time point consistent with influenza-induced depletion ${ }^{29}$. We also examined the total number of TRAIL-positive monocytes, as TRAIL-mediated epithelial cell death has been implicated in influenza-induced lung injury ${ }^{25,26}$. No differences were seen in this cellular compartment either. These data do not exclude potential functional differences in the monocyte compartment as a mechanism for improved bacterial clearance.
IL-22 $\mathrm{BP}^{-/-}$mice have less histologic evidence of tissue inflammation during super-infection

Next, we examined the degree of lung injury induced by superinfection in WT and IL-22BP ${ }^{-/-}$mice. Sections of lung tissue from IL-22BP ${ }^{-1-}$ and WT mice were compared by light microscopy and scored based on the extent of cellular infiltration and tissue damage in the parenchymal, peribronchial, and perivascular regions of the lung. Comparisons of lung injury revealed no significant difference between the IL-22BP ${ }^{-/-}$and WT mice superinfected with influenza, and S. aureus (Fig. 5a). However, damage was noted to be more intense in WT mice while IL-22BP ${ }^{-1-}$ mice had fewer areas of severe damage (Fig. 5c).

$\mathrm{IL}-22 \mathrm{BP}^{-1-}$ mice super-infected with influenza and S. pneumoniae had significantly less lung tissue damage than WT mice (Fig. 5b). The S. pneumoniae super-infection model resulted in worse lung pathology than S. aureus. Notably, WT mice superinfected with influenza and S. pneumoniae showed evidence of extensive tissue inflammation and hemorrhage above and beyond what was observed in super-infected IL-22BP ${ }^{-1-}$ mice (Fig. 5C). Tissue necrosis was also visible on gross examination of the 


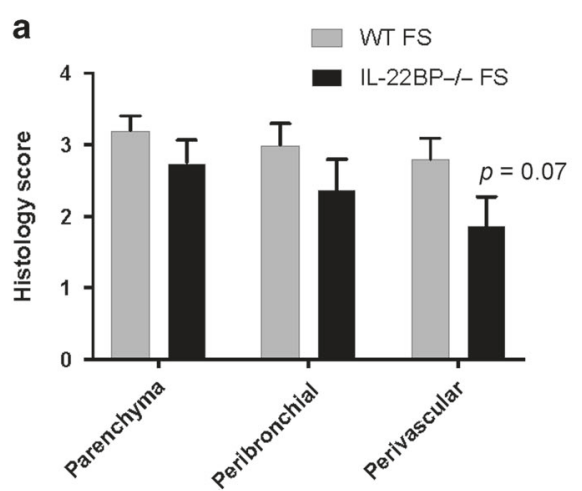

c

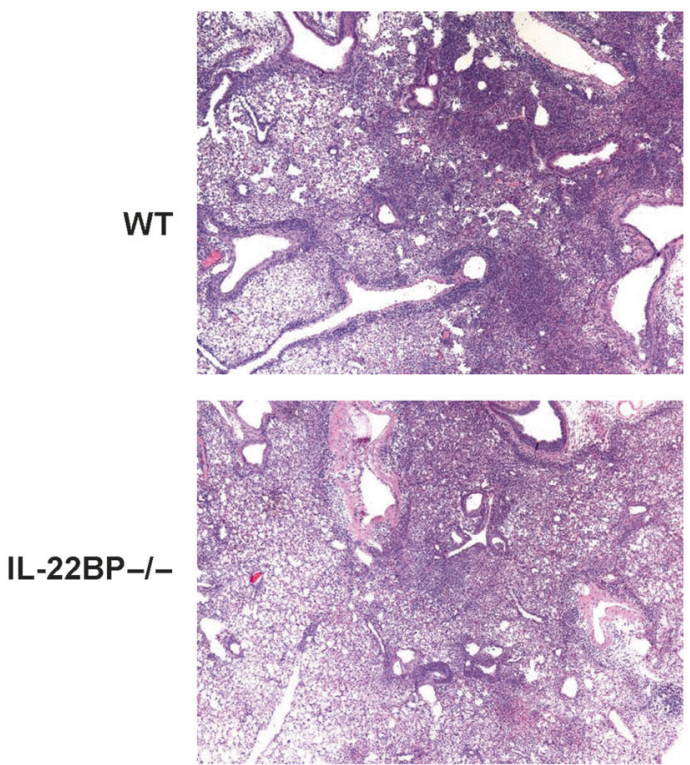

b

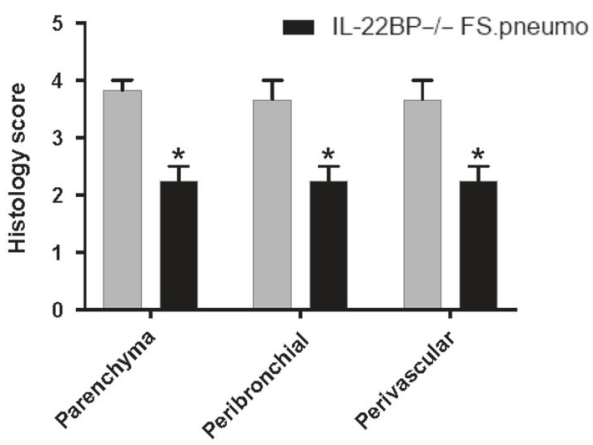

Flu + S. pneumoniae
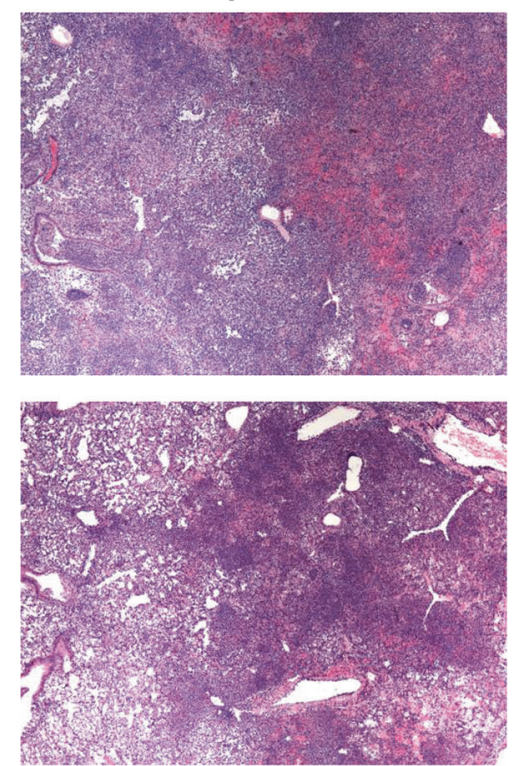

Fig. 5 IL-22BP deficiency alters histopathology during super-infection. C57BL/6 and IL-22BP ${ }^{-/-}$mice were infected with $100 \mathrm{PFU}$ of influenza A/PR/8/34 or vehicle for 6 days prior to infection with $5 \times 10^{7}$ CFU MRSA for $24 \mathrm{~h}$ or $10^{4} \mathrm{CFU} / \mathrm{mL} \mathrm{S}$. pneumoniae for $48 \mathrm{~h}$. a, b Histology score $(n=6-10)$. c Representative hematoxylin-eosin staining of lungs $24 \mathrm{~h}$ after MRSA or $48 \mathrm{~h}$ after $S$. pneumoniae $\left(\times 40\right.$ magnification). ${ }^{*} p<0.05$ Significance was tested by unpaired $t$-test or one-way ANOVA. Each experiment was independently performed twice and the data shown are from combined experiments

harvested lungs of some WT mice (data not shown). These data indicate that IL-22BP ${ }^{-1-}$ mice had ameliorated lung injury during influenza, bacterial super-infection.

IL-22BP ${ }^{-1-}$ mice have decreased epithelial permeability during super-infection

IL-22BP ${ }^{-1-}$ mice super-infected with influenza and S. aureus had a trend towards decreased quantity of total protein in bronchoalveolar lavage (BAL) fluid (Fig. 6a). This finding seemed to suggest a change in the airway epithelium towards reduced permeability. No changes were seen between naïve WT and IL-22BP ${ }^{-1-}$ mice. In order to investigate this further, we injected Evans Blue dye intravenously into the tail vein of WT and IL-22BP ${ }^{-1-}$ mice 30 min prior to lung harvest and measured the leakage of the dye into the BAL fluid spectrophotometrically. The amount of dye in the BAL fluid of IL-22 $\mathrm{BP}^{-1-}$ mice was decreased compared to WT mice confirming that IL-22 $\mathrm{BP}^{-/-}$mice have improved epithelial barrier function (Fig. 6b).

Protein analysis of BAL fluid during influenza, S. pneumoniae super-infected mice were also obtained. Like in the influenza and S. aureus model, there was a trend towards decreased total protein in the BAL fluid of IL-22BP ${ }^{-1-}$ mice compared to WT that did not reach statistical significance (Fig. 6c). Finally, measurement of LDH in BAL fluid revealed no difference in cytotoxic effects between groups (Fig. $6 \mathrm{~d}$ ). In total, these data suggest improved epithelial barrier function in IL-22 $\mathrm{BP}^{-1-}$ mice in the influenza, $S$. aureus model.

Improved tight junction stability is a likely mechanism behind the improved epithelial barrier function in IL-22 $\mathrm{BP}^{-1-}$ mice

We next began to explore potential mechanisms for the protective barrier phenotype observed in IL-22BP ${ }^{-1-}$ mice. Our group and others have previously shown that IL-22 is critical for lung epithelial repair and integrity during influenza $a^{23,30-32}$. We therefore hypothesized that increased IL-22 activity may improve epithelial barrier function and reduced lung leakage in IL-22 $\mathrm{BP}^{-1-}$ mice. The tight junction protein (Tjp) 1 and claudins are proteins involved in tight junction formation and stabilization in many different organ systems ${ }^{33,34}$. We measured Tjp1 and claudin gene expression in influenza, S. aureus super-infected IL-22BP ${ }^{-1-}$ and WT mice, and found that gene expression of Cldn1, Cldn5, and Cldn8 (trend with Cldn4) were significantly increased in the 


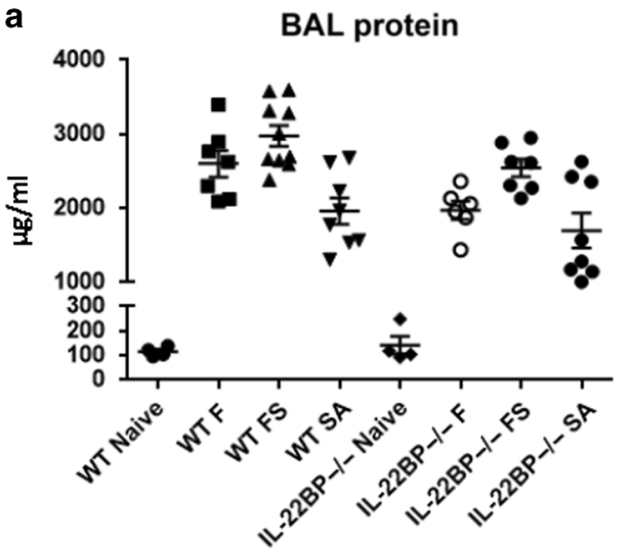

C

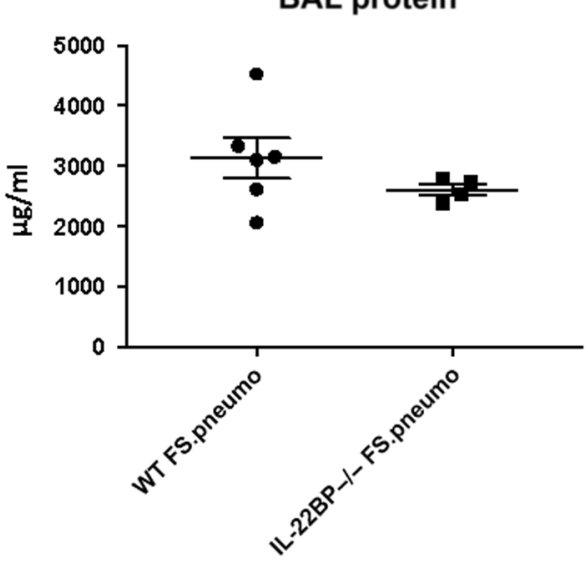

b Evans blue

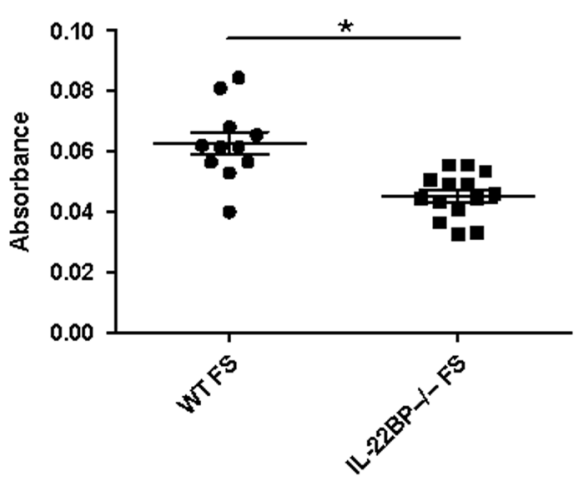

d

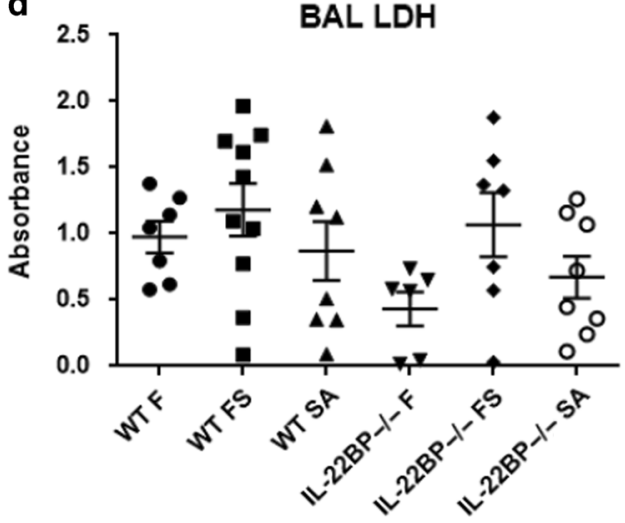

Fig. 6 IL-22BP deletion improves airway epithelial barrier function during super-infection. C57BL/6 and IL-22BP ${ }^{-1-}$ mice were infected with 100 PFU of influenza A/PR/8/34 or vehicle for 6 days prior to infection with $5 \times 10^{7} \mathrm{CFU}$ MRSA for $24 \mathrm{~h}$ or $10^{4} \mathrm{CFU} / \mathrm{mL} \mathrm{S}$. pneumoniae for $48 \mathrm{~h}$. a c Protein in BAL fluid ( $n=4-10)$. b Absorbance of Evans Blue dye in BAL fluid $(n=11-14)$. d Absorbance of lactate dehydrogenase in BAL fluid $(n=6-10) .{ }^{*} p<0.05$ Significance was tested by unpaired $t$-test or one-way ANOVA. Each experiment was independently performed twice and the data shown are from combined experiments, except for panel e which is a single representative study

IL-22BP ${ }^{-1-}$ mice compared to WT (Fig. 7a). No significant changes were seen in epithelial junctional protein expression during influenza infection alone (Fig. 7b). These data combined with pulmonary leak data suggest that IL-22 $\mathrm{BP}^{-/-}$mice are protected by increased epithelial barrier integrity.

To examine a potential effect of IL-22 on epithelial barrier integrity, we used an air-liquid interface epithelial model to evaluate the effects of IL-22Fc treatment on human bronchial epithelial cells (HBEs). HBEs were challenged with S. aureus for $24 \mathrm{~h}$ in the presence or absence of IL-22Fc. Tight junction integrity was measured by transepithelial electrical resistance (TEER). Resistance was maintained at a higher level in the IL-22Fc-treated group compared to untreated cells providing evidence for superior barrier integrity (Fig. 7c). To examine molecular mechanisms that underlie the improved junctional integrity, we used immunofluorescence microscopy to observe the association between occludin-1, a protein essential for tight junction stability, to the tight junction-associated protein zonula occludens-1 (ZO-1) in IL$22 \mathrm{Fc}$-treated cells. We found increased localization of ZO-1 with occludin-1 in IL-22Fc-treated cells compared to the untreated cells (Fig. 7d, e). These data confirm a role for IL-22 in the maintenance of tight junction stability and barrier function.

Potential mechanism for increased bacterial clearance in IL-22 $\mathrm{BP}^{-/-}$ mice during super-infection

First, we examined whether $1 / 22 b p$ is differentially expressed in super-infection versus single infection. Interestingly, $1122 b p$ expression was significantly lower during influenza or superinfection compared to $S$. aureus single infection (Fig. 8a). These data are consistent with the decrease in $1122 b p$ observed over time during influenza infection. A potential mechanism by which IL-22 may exert protective function in IL-22BP ${ }^{-1-}$ mice would be altered II22ra1 expression. However, II22ra1 expression was not different between WT and IL-22BP ${ }^{-1-}$ mice during single or super-infection (Fig. 8b).

We have previously shown that $/ 117$ and $/ 122$ expressions are decreased in super-infection compared to $S$. aureus single infection $^{22}$. This was later associated with defective AMP production in super-infection ${ }^{35}$. IL-10 was shown to limit Type 17 responses and increase bacterial burden in this model ${ }^{36}$. To determine if IL-22BP deletion impacts these known pathways we examined each. WT and IL-22BP ${ }^{-/-}$mice had similar expression of $I 122$ and $/ 117$ (Fig. 8c, d). The lack of change in $1 / 22$ expression in $\mathrm{IL}-22 \mathrm{BP}^{-1-}$ mice does not exclude the possibility of increased IL22 in the absence of its decoy receptor. IL-10 protein levels trended to be decreased in the lungs of IL-22BP ${ }^{-1-}$ compared to WT mice (Fig. 8e). We then measured AMP expression in the lungs of WT and IL-22BP ${ }^{-1-}$ mice during influenza alone or super-infection. Lipocalin 2 expression was higher in IL-22BP ${ }^{-1-}$ compared with WT mice during super-infection (Fig. 8f). There were no changes in AMP expression between WT and IL-22 $\mathrm{BP}^{-/-}$ mice during influenza infection alone. We and others have previously shown that lipocalin 2 promotes $S$. aureus clearance in super-infection models ${ }^{35,37}$. These data suggest that improved 
a
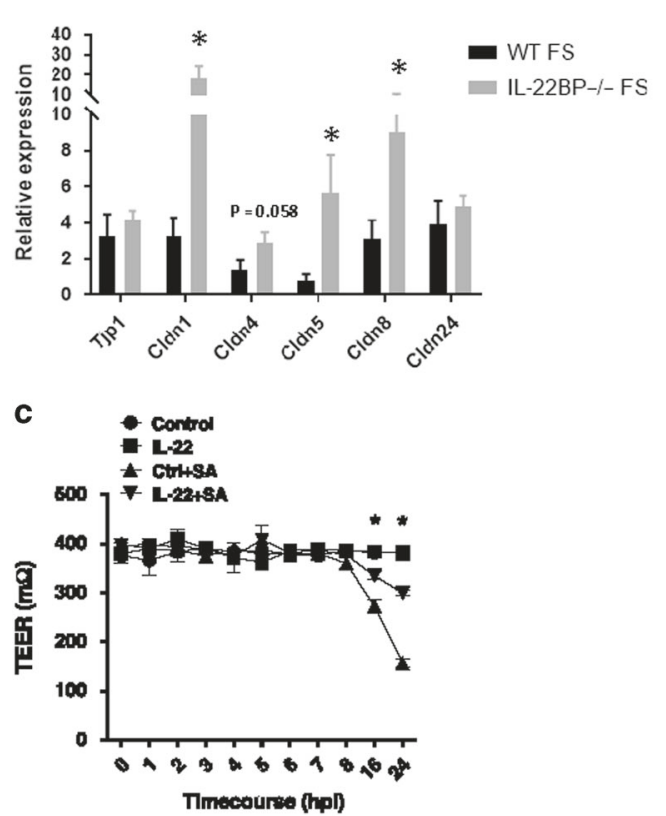

b Junction proteins

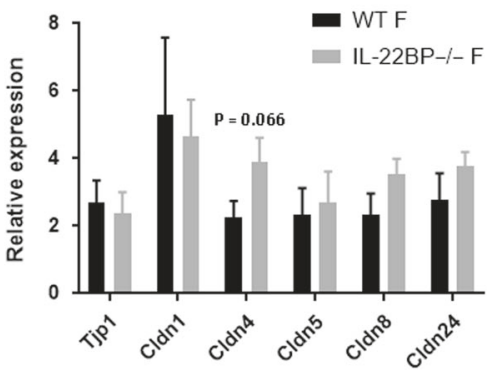

d ZO-1

Occludin

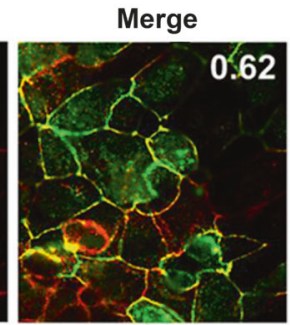

Control
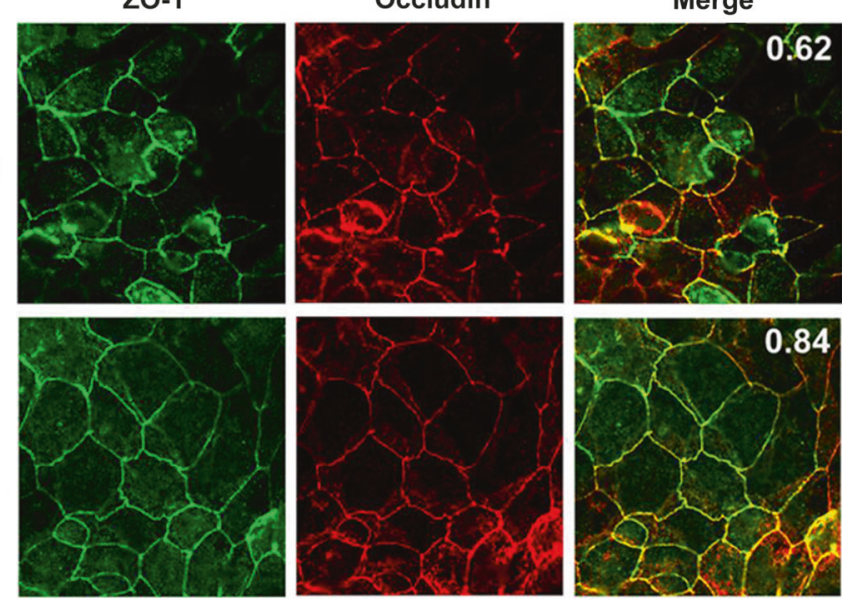

e
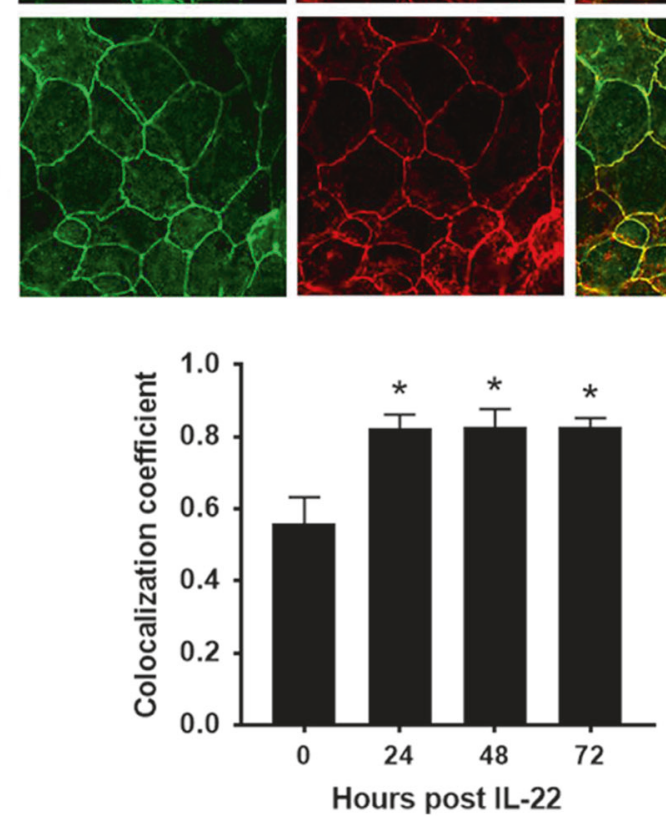

Fig. 7 IL-22BP and IL-22 are important for airway epithelial tight junction integrity. $\mathbf{a}, \mathbf{b}$ Claudin and Tjp1 gene expression measured by RT-PCR in C57BL/6 and IL-22BP ${ }^{-1-}$ mice that were infected with 100 PFU of influenza A/PR/8/34 for 6 days prior to infection with $5 \times 10^{7}$ CFU MRSA for $24 \mathrm{~h}(\mathbf{a}, n=8-10)$ or influenza alone (b, $n=6-9)$. c Transepithelial electrical resistance of air-liquid differentiated human bronchial epithelial cells at 0 through $24 \mathrm{~h}$ after inoculation with $\mathrm{S}$. aureus and treatment with IL-22Fc or vehicle ( $n=3$ independent cultures). d Immunofluorescence staining of polarized human bronchial epithelial cells treated with IL-22Fc or vehicle for $24 \mathrm{~h}$. Inset numbers reflect ratio of overlap staining. e Colocalization of ZO- 1 and occluden in cells treated with IL-22Fc for $0-72 \mathrm{~h}$ ( $n=3$ independent cultures, six fields per culture). ${ }^{*} p<0.05$ Significance was tested by unpaired $t$-test or two-way ANOVA. Each experiment was independently performed two to three times and the data shown are from combined experiments except for panel $\mathbf{d}$ which is a single representative study 
a

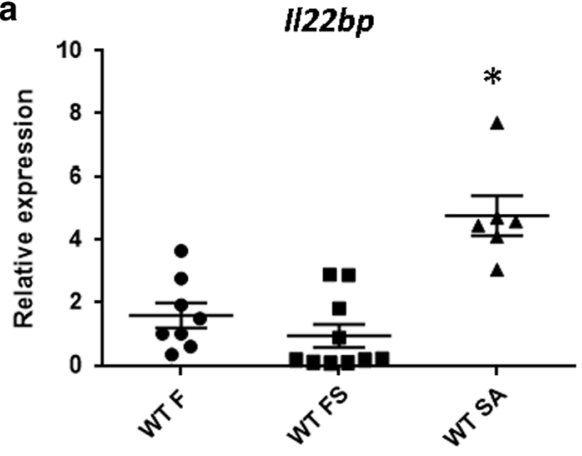

C

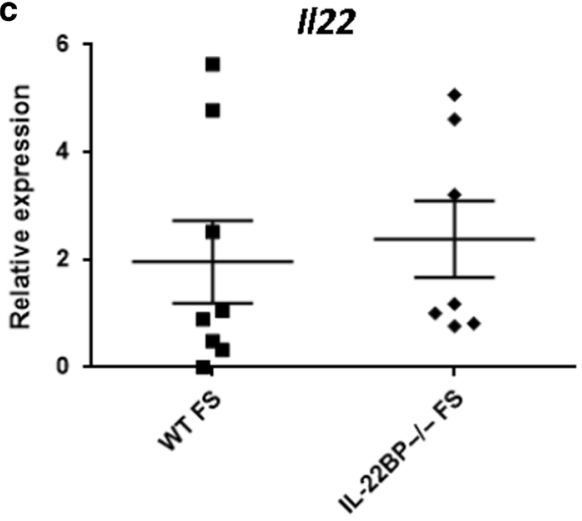

e

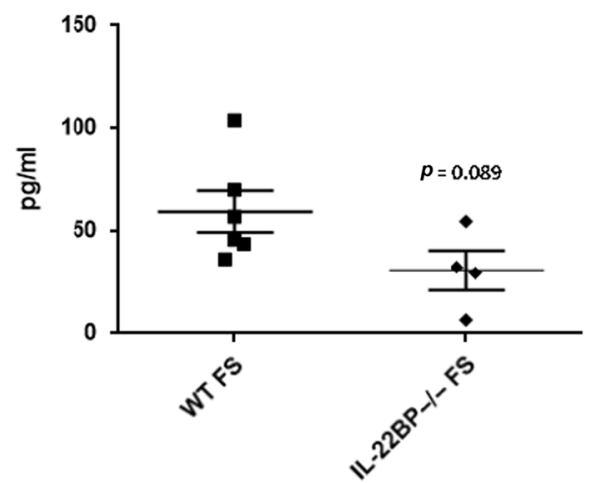

b

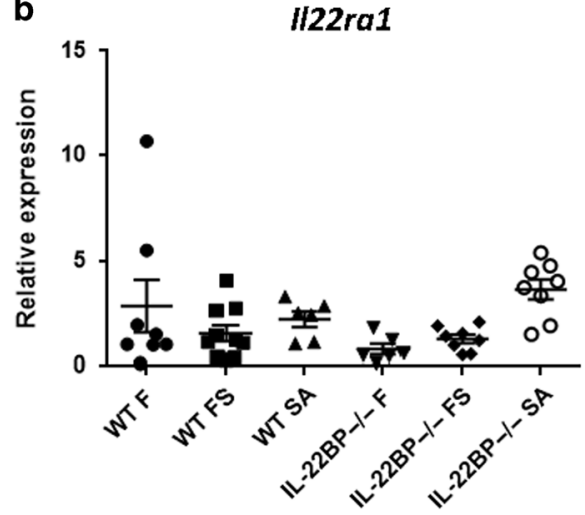

d

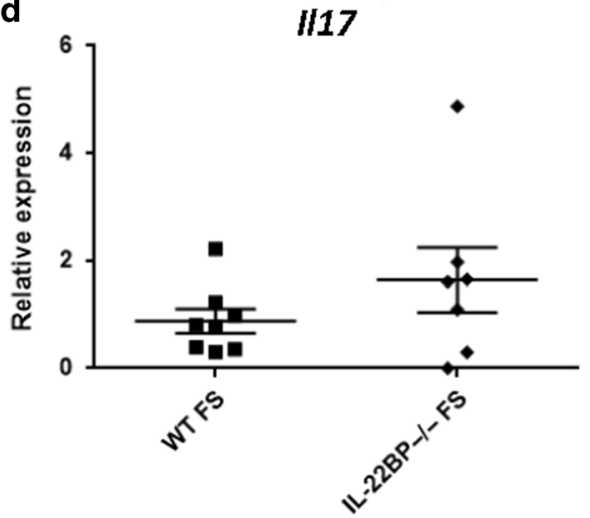

f

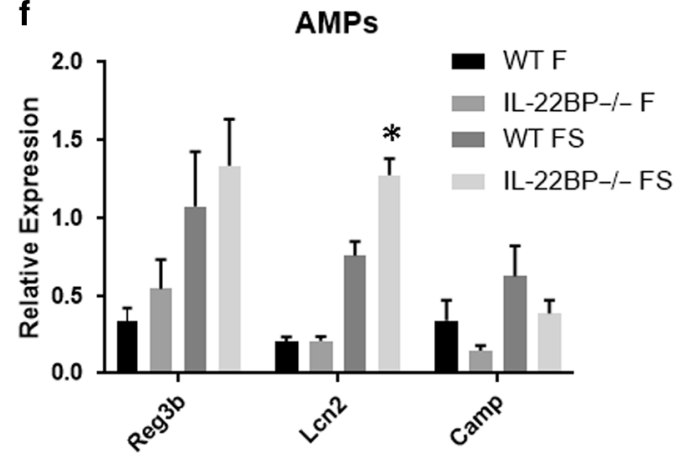

Fig. 8 Deletion of IL-22BP results in increased lipocalin 2 expression. C57BL/6 and IL-22BP ${ }^{-1-}$ mice were infected with 100 PFU of influenza A/PR/8/34 or vehicle for 6 days prior to infection with $5 \times 10^{7}$ CFU MRSA for $24 \mathrm{~h}$. a $/ 122 \mathrm{bp}$ expression during single or super-infection $(n=$ 6-10). b $/ 122 \mathrm{ra} 1$ expression in WT and IL-22BP ${ }^{-1-}$ mice $(n=6-10)$. c, d $/ 117$ and $/ 122$ expression during super-infection ( $\left.n=7-8\right)$. e IL-10 protein concentration in lung homogenate $(n=4-6)$. f AMP expression during influenza single infection or super-infection $(n=4-6)$. Significance was tested by unpaired $t$-test or two-way ANOVA. Each experiment was independently performed twice and the data shown are from combined experiments

bacterial clearance in IL-22 $\mathrm{BP}^{-1-}$ mice may be due to increased lipocalin 2 expression.

\section{DISCUSSION}

Few studies have examined the role of IL-22BP in lung infection and, to our knowledge, this study is the first to identify an IL-22BP effect in the context of bacterial super-infection of the lungs. Secondary bacterial pneumonia is a major contributor to influenza-related mortality and we have shown that IL-22BP is a critical negative regulator of secondary infection by both $S$. aureus and S. pneumoniae following influenza. When IL-22BP is absent, mice are protected from super-infection with decreased bacterial burden, decreased inflammation, and preservation of epithelial barrier integrity. Together these alterations in the disease characteristics provide a survival benefit to IL-22BP ${ }^{-1-}$ mice during super-infection. Interestingly, IL-22 $\mathrm{BP}^{-1-}$ mice did not have a bacterial clearance benefit in $S$. aureus single infection. This is likely explained by the higher levels of IL-22 present in this setting compared to super-infection. IL-22BP may not be a critical regulator of IL-22 when IL-22 levels are highly elevated. Alternatively, influenza may create an immune setting, where IL22 is more critical to $S$. aureus clearance and thus IL-22BP's role is enhanced in super-infection. The ability of IL-22BP deletion to ameliorate super-infection in two bacterial models is an important consideration. S. aureus does not replicate well in the mouse lung, 
whereas S. pneumoniae does. IL-22BP ${ }^{-1-}$ mice had improved phenotypes in both high dose bacterial challenge (S. aureus) and low dose infection (S. pneumoniae). This finding strengthens the potential role of IL-22BP in disease pathogenesis.

First, we examined the effect of influenza infection on the IL-22 pathway. Influenza infection increased IL-22 production in the lung with a peak at day 6 post-infection. During the same time frame IL-22Ra1 and IL-22BP expression is decreased below naïve levels ( $50 \%)$, although expression of each remains readily detectable. This correlates with the timing of heightened susceptibility to secondary bacterial infection. Deletion of IL-22BP ${ }^{-1-}$ did not impact influenza-induced weight loss or cellular inflammation in the BAL. There was a trend towards an increase in viral burden and a trend towards decreased BAL protein leak in IL-22 $\mathrm{BP}^{-/-}$versus WT mice during single influenza infection. These data suggest that the role of IL-22BP during acute influenza single infection is less robust than during superinfection. We have previously shown that IL-22 plays a critical role in lung repair during influenza infection with the effect observed at time points later than those studies herein ${ }^{32}$. It is likely that IL-22BP has an impact at later time points during influenza infection resolution. We did observe increased viral burden in IL$22 \mathrm{BP}^{-1-}$ mice during super-infection. This may be the result of decreased epithelial cell death and thus higher viral replication; however, we do not know the mechanism for this result.

IL-22BP is a soluble decoy receptor with a high affinity for IL-22. Binding by IL-22BP neutralizes the effect of IL-22 by preventing it from binding to its cell surface receptor. This inhibitory role of IL22BP has been previously shown to be important in a variety of inflammatory disease states including those of skin and gastrointestinal tract. Although the effect of IL-22 may be positive or negative depending on the disease state, in all instances IL-22BP has been associated with close regulation of IL-22 activity ${ }^{15}$.

Investigation into the source of IL-22BP is ongoing with most studies focusing on cellular sources within the gut. Recent studies have found evidence of IL-22BP expression in the spleen, lymph nodes, and epithelial tissues of the lungs, skin, and intestines. Specifically, they have shown evidence of IL-22BP expression by subsets of dendritic cells ${ }^{38,39}$ as well as $\mathrm{CD}^{+}{ }^{+} \mathrm{T}$ cells and eosinophils in the small intestine and lymph nodes ${ }^{38}$.

In the lung IL-22BP appears to exacerbate the highly inflammatory response to super-infection. When absent there is less inflammation visualized microscopically and reduced influx of both pro-inflammatory cytokines and neutrophils across the epithelial barrier. The result is a stark contrast of uncontrolled inflammation and infection in WT mice and a more regulated host response in IL-22BP ${ }^{-1-}$ mice. Unlike the influenza, S. pneumoniae model, we did not observe a significant decrease in lung pathology in the influenza, S. aureus model. This may be due to the high inoculum of bacteria in that model. Overall, these results are consistent with previous studies that associate uncontrolled or atypical inflammatory responses with increased, morbidity and mortality from influenza and secondary bacterial pneumonia ${ }^{40,41}$. It is therefore no surprise that super-infected WT mice exhibit higher disease burden compared to IL-22 $\mathrm{BP}^{-/-}$mice during super-infection.

The association we observed between IL-22BP and increased inflammation is in agreement with previous studies from other organ systems as well. These include a number of studies focusing on IL-22BP in the setting of inflammatory diseases of the intestines and liver. A recent study found that IL-22BP is produced by $\mathrm{CD}^{+}$ $T$ cells in a mouse model of inflammatory bowel disease (IBD) and appears to be essential for the development of the inflammation that defines the disease. This study also revealed that treatment of mice with IBD with an anti-TNF-a agent reduced IL-22BP expression suggesting that some of the anti-inflammatory effects of the treatment may be a result of a down-regulating effect on IL$22 \mathrm{BP}^{42}$. We did not observe a difference in TNF-a protein levels in
WT and IL-22BP ${ }^{-1-}$ super-infected mice (data not shown). A separate study examined the effect of IL-22BP injections into the proximal colon in a mouse model of ulcerative colitis to evaluate the effect on disease process. Inflammation in the area of injection was notably increased in IL-22BP-injected mice compared to those that received a mock injection ${ }^{43}$.

In the liver, chronic inflammation is a primary driver for the development of fibrosis and cirrhosis, and IL-22BP has been implicated in the regulation of fibrosis and cirrhosis in chronic hepatitis $C$ disease based on an association with IL-22BP gene variants $^{19}$. However, a study on acute liver injury by ischemic reperfusion and acetaminophen administration showed contrasting results. It identified a protective effect of IL-22BP with evidence of increased inflammatory monocyte infiltration in IL22BP-deficient mice ${ }^{44}$. This may suggest an effect of IL-22BP in the liver that is in opposition to what has been described in respiratory and intestinal inflammatory diseases. This may be explained in part by the dual role of IL-22 depending on the disease context and the overall need for tightly regulated IL-22 activity $^{45}$.

Similar effects of IL-22BP have been observed in inflammatory disorders of the nervous system. IL-22BP has been identified as a risk gene for MS and has been studied in the experimental autoimmune encephalomyelitis (EAE) mouse model, where it was found to have a pro-inflammatory role in the central nervous system. The study found that IL-22 $\mathrm{BP}^{-1-}$ mice had less severe disease with reduced spinal cord inflammation consistent with what we observed in the super-infected lungs of IL-22 $\mathrm{BP}^{-1-}$ mice. In contrast to our study, they found no differences in neutrophil accumulation between the IL-22BP ${ }^{-1-}$ and control groups ${ }^{18}$. These differences are likely justified by the distinctions between autoimmune inflammatory diseases and infectious disease processes.

Psoriasis is a disease where the pro-inflammatory role of IL-22 has been well-described as a contributor to epithelial remodeling and inflammation. Two recent studies have identified an inflammation-modulating role for IL-22BP in psoriasis, albeit an anti-inflammatory effect given the opposing role of IL-22 in the disease. The first examined a rat model of imiquimod-induced skin disease and identified increased inflammatory changes on clinical and histologic examination in IL-22 $\mathrm{BP}^{-1-}$ rats. In addition, IL$22 \mathrm{BP}^{-1-}$ rats had increased expression of pro-inflammatory cytokines compared to those with preserved IL-22BP ${ }^{46}$. The second study examined a similar imiquimod-induced skin disease in mice as well as skin biopsies of patients with and without psoriatic skin disease. They noted reduced expression of IL-22BP in mouse-model as well as in patients with psoriasis. They concluded that regulation of IL-22BP is a key step in the development of skin inflammation ${ }^{47}$. While the effect of IL-22BP is reversed in skin disease, its ability to regulate inflammation through action on IL22 is consistent with our findings.

Delving into the potential mechanisms of protection from mortality in super-infected IL-22 $\mathrm{BP}^{-1-}$ mice revealed a notable change in barrier function of the airway mucosal layer. A fundamental aspect of host defense in this region is the ability to provide a barrier against pathogens from the external environment. Impairment raises the infection risk and can potentially lead to increased disease severity. Our study revealed that the absence of IL-22BP was associated with reduced permeability of the airway epithelium and markedly improved barrier function. The results were consistent between our influenza, S. aureus and influenza, S. pneumoniae models of super-infection that both demonstrated decreased passage of inflammatory cells and protein into the BAL fluid of IL-22BP ${ }^{-1-}$ mice. This superior barrier integrity may be beneficial for host defense against bacterial pathogens. First, it may limit bacterial spread from the airway lumen into the lung parenchyma therefore allowing the infection to be better contained. We did not observe 
a significant amount of bacterial dissemination from the lungs to other organs in the influenza, S. aureus model and dissemination was not altered in IL-22 $\mathrm{BP}^{-1-}$ compared with WT mice in the pneumococcal model. These data indicate that bacterial dissemination to other organs does not likely contribute to mortality in these models. We cannot exclude the possibility that epithelial barrier function dictates bacterial localization in the lung itself, which is likely. Further, IL-22BP ${ }^{-1-}$ mice may have altered bacterial dissemination in models where bacterial escape from the lung is more common. Second, barrier integrity may limit the degree of uncontrolled inflammation that occurs through the influx of inflammatory mediators that would otherwise tend to exacerbate disease course. Influx of recruited monocytes to the lung was not different in WT and IL-22 $\mathrm{BP}^{-1-}$ mice, although the localization of these cells was not determined. It has been shown previously that in the Gl tract, at least some of IL-22BP's effect appears to be via inhibition of the epithelial protective effects of IL-22 on the intestinal lining ${ }^{42}$. This could explain our findings based on the hypothesis that in the lungs IL-22BP regulates IL-22 in a similar fashion.

In addition to alterations in epithelial protective effects, we have shown evidence in our influenza, S. aureus super-infection model that the improvement in barrier function in IL-22 $\mathrm{BP}^{-1-}$ mice is related to the maintenance of tight junctions. Specifically, the absence of IL-22BP is associated with increased gene expression of claudins $1,4,5$, and 8 , barrier forming tight junction proteins. Claudins are the largest family of tight junction proteins and are expressed by multiple cell types throughout the airways ${ }^{33,34}$. Influenza infection has been shown to decrease expression of claudins 1 and 4 in human epithelial cell culture models resulting in decreased tight junctions ${ }^{48,49}$. Increased claudin expression in $\mathrm{IL}-22 \mathrm{BP}^{-1-}$ mice may contribute to the reduced passage of substances across the airway epithelium during super-infection. This is supported by our observations that pro-inflammatory cytokines and neutrophil accumulation are both reduced in the $\mathrm{BAL}$ fluid of IL-22BP ${ }^{-1-}$ mice. Further study is necessary to determine whether the effect of IL-22BP on tight junction function is related to IL-22 activity.

IL-22 is also known to contribute to barrier function and has been shown to be involved in the maintenance and repair during influenza and bacterial infection ${ }^{30-32,50}$. Our results suggest that the mechanism of barrier preservation by IL-22 may be an effect on tight junctions similar to what was observed in the superinfected IL-22BP ${ }^{-1-}$ mice. HBECs treated with IL-22Fc had increased TEER compared to untreated cells and this resistance is maintained primarily by the integrity of tight junctions. This is indicative of enhanced function of tight junctions with IL-22Fc treatment. Immunofluorescence staining furthermore showed that IL-22Fc treatment increased the localization of ZO-1 to occludin at tight junctions, a necessary step to assure tight junction stability. IL-22Fc has recently been shown to be an effective treatment in a mouse model of influenza, $S$. pneumoniae super-infection. Treatment with IL-22Fc was associated with increased epithelialadhesion protein, extracellular matrix, and epithelial cell proliferation gene expression that was suggestive of improved barrier function. Treated mice also had decreased lung pathology and reduced dissemination of $S$. pneumoniae to the spleen ${ }^{23}$. These findings of improved barrier function with IL-22 supplementation are consistent with what we observed in vitro. The concept of regulation of tight junction proteins by $\mathrm{IL}-22$ is consistent with previous studies evaluating non-respiratory organ systems. Within the gastrointestinal tract, IL-22 has been shown to induce gene expression of claudin-2, a pore forming tight junction protein that, among other functions, increases intestinal permeability in IBD ${ }^{51}$.

The role of Type 17 immunity in bacterial clearance of $S$. aureus in the context of influenza super-infection has been demonstrated $^{52}$. We have previously shown that preceding influenza attenuates IL-17 and IL-22 and subsequent AMP production in response to $S$. aureus infection ${ }^{22,35}$. Further $\mathrm{IL}-10^{-1-}$ mice displayed increased Type 17 immune activation during superinfection indicating its detrimental role in this mode ${ }^{36}$. We first examined whether IL-22BP deletion impacts IL-17 or IL-22 gene expression. We did not see significant changes in either cytokine in comparison with WT mice. However, this finding does not exclude the possibility that IL-22 has enhanced activity in the lungs of IL-22BP $\mathrm{BP}^{-1-}$ mice. This potential effect is supported by increased expression of lipocalin 2 in IL-22BP ${ }^{-1-}$ compared to WT mice during super-infection, but not during single influenza infection, where lipocalin 2 expression was lower than superinfected mice. Our group and others have shown that small magnitude changes in lipocalin 2 expression is associated with improved bacterial control in influenza, super-infection ${ }^{35,37}$. In that study, we showed that while IL-22 treatment of mouse airway epithelial cells did not induce lipocalin 2, IL-22 did further enhance IL-17 and TNFa-induced lipocalin 2 expression. It is possible that this increase in lipocalin 2 is responsible for the improved bacterial clearance observed, likely through regulation of iron availability.

In conclusion, this study has demonstrated the detrimental, proinflammatory role of IL-22BP during influenza, bacterial superinfection of the lungs. This expands upon the current body of literature that has identified similar roles for IL-22BP in other inflammatory diseases. Furthermore, we have identified a novel protective effect of IL-22BP deletion on super-infection. Improved tight junction function and lipocalin 2 production and reduced membrane permeability may represent possible mechanisms behind this protection and warrant additional study to evaluate IL-22BP as a therapeutic target. In humans, there are three IL-22BP isoforms present with only IL-22BPi2 likely to have IL-22-binding activity in the context of infection ${ }^{53}$. Further study is required to determine the potential efficacy of IL-22BP antagonism in disease.

\section{METHODS}

Mice

Six to eight-week-old male C57BL/6 mice were purchased from Taconic Farms (Germantown, NY). IL-22BP ${ }^{-1-}$ mice backcrossed 9 times onto the C57BL/6 background were obtained from Genentech (San Francisco, CA) ${ }^{38}$. Mice were maintained under pathogen-free conditions and studies were performed on age and sex-matched mice.

\section{S. aureus infection}

MRSA (USA 300) was propagated as previously described ${ }^{54}$. Mice were inoculated with MRSA by oropharyngeal aspiration. Mice received doses of either $2.5 \times 10^{8}$ or $5 \times 10^{7}$ colony-forming units of MRSA (in $50 \mu \mathrm{L}$ sterile phosphate-buffered saline (PBS)) during the survival study and all other studies, respectively.

\section{S. pneumoniae infection}

One milliliter of a stock of S. pneumoniae serotype 3 (ATCC 6303) was grown in $5 \mathrm{~mL}$ of Todd-Hewitt broth for $6 \mathrm{~h}$ at $37^{\circ} \mathrm{C}, 5 \% \mathrm{CO}_{2}$. A $100 \mu \mathrm{L}$ aliquot of the solution was then inoculated into $100 \mathrm{~mL}$ of Todd-Hewitt broth and grown for an additional $12 \mathrm{~h}$. The concentration was determined by measuring the absorbance at $600 \mathrm{~nm}$ with an optical density of 0.5 corresponding to a concentration of $1 \times 10^{8} \mathrm{CFU} / \mathrm{mL}$. Mice were inoculated with $10^{4}$ $\mathrm{CFU} / \mathrm{mL}$ (in $50 \mu \mathrm{L}$ sterile PBS) by oropharyngeal aspiration. The stock was serially diluted and plated on trypticase soy agar with $5 \%$ sheep blood plates to confirm the concentration given.

Influenza $A$ and super-infection

Influenza A PR/8/34 H1N1 was propagated as previously described $^{55}$. Mice were inoculated with 100 plaque-forming units (PFU) of influenza A PR/8/34 H1N1 (in $40 \mu \mathrm{L}$ sterile PBS) by oropharyngeal aspiration. Six days later mice were infected with 
1242

bacteria or PBS vehicle as described and were harvested 24 or $48 \mathrm{~h}$ later. For time course studies, mice were harvested every 2 days following infection for 2 weeks. Weight loss induced by influenza or super-infection was tracked by the percentage of loss compared to original starting weight of each mouse.

\section{Epithelial permeability}

Mice were injected with $20 \mathrm{mg} / \mathrm{kg}$ of Evans Blue dye (Sigma, St. Louis, MO) via the dorsal tail vein 30 min prior to lung harvesting and BAL fluid was obtained as described below. BAL fluid was centrifuged and the absorbance of $100 \mu \mathrm{L}$ undiluted supernatant was measured at $630 \mathrm{~nm}$ using a linear standard curve.

\section{Analysis of lung infection}

Mouse lungs were lavaged with $1 \mathrm{~mL}$ sterile PBS for inflammatory cell counts. Cytospin preparations of BAL fluid were stained using Diff-Quick (Sigma, St. Louis, MO) and differential cell counts were conducted based on cell morphology. The superior lobe of the right lung was homogenized in sterile PBS $(1 \mathrm{~mL})$ by mechanical grinding. The lung homogenate was use for bacterial colony counting cytokine analysis by Bio-plex Multiplex immunoassay (BioRad, Hercules, CA). Middle and inferior lobes of the right lung were snap-frozen and homogenized under liquid nitrogen for RNA extraction using an RNA isolation kit (Agilent Technologies, Santa Clara, CA). RNA analysis was performed by RT-PCR using Assay on Demand TaqMan probes and primers (Applied Biosystems, Foster City, CA). For bacterial dissemination studies, the liver, spleen, and kidneys were homogenized in PBS and plated for colony counts. Influenza burden was determined by RT-PCR for matrix protein expression as previously described ${ }^{56}$.

\section{Histology scoring}

A cold, $10 \%$ neutral buffered formalin solution (Sigma, St. Louis, MO) was used for fixation of the left lungs. Lungs were inflated and immersed in the formalin solution prior to storage. Slides were then prepared by Histo-Scientific Research Laboratories (Mount Jackson, VA) and blindly reviewed and scored using a validated histology scoring system that has been previously described ${ }^{57}$. Each slide received three scores from 1 to 4 based on the degree of cellular infiltration and tissue damage in the parenchymal, peribronchial, and perivascular regions.

In vitro cell culture technique

The role of IL-22 in barrier integrity was studied in human airway epithelial cells (16HBE cells). The derivation and characterization of these cells have been described in detail ${ }^{58}$. Airway epithelial cells between passages 45 and 55 were grown and polarized in an air-liquid interface culture at $37^{\circ} \mathrm{C}$ for 6-9 days, as described previously ${ }^{59}$.

Transepithelial electrical resistance

TEER was measured on air-liquid differentiated HBEs (16HBE) at 0 through $24 \mathrm{~h}$ after inoculation with S. aureus (MRSA; USA 300) and treatment with IL-22Fc or vehicle, as described previously ${ }^{59}$.

Immunofluorescence staining and microscopy

Confocal microscopy was performed, as described previously ${ }^{60}$ to visualize the localization of junctional proteins in response to IL-22 Fc treatment, polarized $16 \mathrm{HBE}$ cells were treated basolaterally with IL-22 Fc for $24 \mathrm{~h}$ at $37^{\circ} \mathrm{C}$. Cells were washed with PBS and fixed with $4 \%$ paraformaldehyde, followed by permeabilization with $0.25 \%$ Triton X-100 in PBS and incubation with the indicated primary antibodies for $1 \mathrm{~h}$ at room temperature. The cells were then washed, incubated with secondary antibodies for $30 \mathrm{~min}$ at room temperature, washed, and mounted with ProLong Gold (Invitrogen). Images were captured using an FV1000 confocal laser scanning microscope (Olympus) and merged and colocalization measured using Nikon Elements software. Antibodies or other reagents for fluorescence microscopy were as follows: rabbit antiZO-1 (mid-region; Invitrogen), mouse anti-occluding-1 (Invitrogen). Experiments were repeated three times, with six fields imaged for each experiment.

\section{Flow cytometry}

To obtain a single-cell suspension, lungs were mechanically dissected, and then incubated shaking at $37^{\circ} \mathrm{C}$ for $30 \mathrm{~min}$ in DMEM containing $10 \%$ FBS and $1 \mathrm{mg} / \mathrm{mL}$ collagenase. After collagenase treatment, tissue was forced through a $70 \mu \mathrm{M}$ filter and treated with ACK buffer to lyse erythrocytes. The resulting single-cell suspension was pre-treated with anti-CD16/32 for 5 min to block Fc receptor binding before incubating with fixable viability dye and fluorochrome-conjugated anti-surface marker monoclonal antibodies for $30 \mathrm{~min}$ at $4{ }^{\circ} \mathrm{C}$. The following antibodies were used: anti-CD45, anti-CD11b, anti-CD11c, anti-CD64, anti-SiglecF, antiLy6g, and anti-MHCII. LIVE/DEAD Fixable Aqua Stain (Life Technologies, Carlsbad, CA) was used to determine cell viability. Samples were collected using a LSRFortessa flow cytometer (BD Biosciences, San Jose, CA) and analyzed using FlowJo software (vX.0.7, Tree Star, Ashland, OR). Flow gating began with doublet exclusion by comparing forward light scatter area versus height, then debris exclusion by comparing forward versus side light scatter. Dead cells were excluded based on viability dye staining. Within the CD45 positive, Ly6g negative gate inflammatory

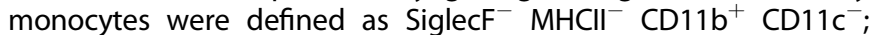

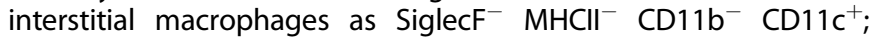
monocyte-derived dendritic cells as SiglecF ${ }^{-} \mathrm{MHClI}^{+} \mathrm{CD}_{11 \mathrm{~b}^{+}}$. Alveolar macrophages were defined as $\mathrm{CD}_{4} 5^{+}$SiglecF $^{+}$ $\mathrm{CD}^{+}{ }^{+}$cells.

\section{Statistics}

All of the data are presented as the mean \pm SEM. Significance was tested by the unpaired $t$-test (for two means) or one-way ANOVA followed by Tukey test (for multiple data groups). The Gehan-Brislow-Wilcoxon test was used for survival data analysis. All data were analyzed using GraphPad Prism software.

\section{Study approval}

All animal experiments were conducted with approval from the University of Pittsburgh Institutional Animal Care and Use Committee.

\section{ACKNOWLEDGEMENTS}

This work was supported by NIH NHLBI R01HL107380 (J.F.A.), Pennsylvania Department of Health (J.F.A.), NIDDK P30DK072506 (J.F.A., J.M.B.), and T32HL129949 (R.N.A.).

\section{AUTHOR CONTRIBUTIONS}

J.F.A., J.M.B., J.K.K., D.A.P., K.M.R. conceived the studies. R.N.A., K.J.M., H.E.R., K.R., J.M.B., M.L.M., M.A.O., J.M.T. performed the studies. R.N.A., J.M.B., J.F.A. wrote the manuscript. FundingThis work was supported by NIH NHLBI R01HL107380 (J.F.A.), Pennsylvania Department of Health (J.F.A.), NIDDK P30DK072506 (J.F.A., J.M.B.), and T32HL129949 (R.N.A.).

\section{ADDITIONAL INFORMATION}

Competing interests: The authors declare no competing interests.

Publisher's note: Springer Nature remains neutral with regard to jurisdictional claims in published maps and institutional affiliations.

\section{REFERENCES}

1. Rolfes, M. A. et al. Effects of influenza vaccination in the United States during the 2017-2018 influenza season. Clin. Infect. Dis. (2019). https://doi.org/10.1093/cid/ ciz075. [Epub ahead of print]. 
2. Centers for Disease Control and Prevention (CDC). Bacterial coinfections in lung tissue specimens from fatal cases of 2009 pandemic influenza A (H1N1) - United States, May-August 2009. MMWR Morb. Mortal. Wkly. Rep. 58, 1071-1074 (2009).

3. Morens, D. M., Taubenberger, J. K. \& Fauci, A. S. Predominant role of bacterial pneumonia as a cause of death in pandemic influenza: implications for pandemic influenza preparedness. J. Infect. Dis. 198, 962-970 (2008).

4. Shieh, W. J. et al. 2009 pandemic influenza $A$ (H1N1): pathology and pathogenesis of 100 fatal cases in the United States. Am. J. Pathol. 177, 166-175 (2010).

5. Finelli, L. et al. Influenza-associated pediatric mortality in the United States: increase of Staphylococcus aureus coinfection. Pediatrics 122, 805-811 (2008).

6. Rice, T. W. et al. Critical illness from 2009 pandemic influenza A virus and bacterial coinfection in the United States. Crit. Care Med 40, 1487-1498 (2012).

7. Williams, D. J. et al. Influenza coinfection and outcomes in children with complicated pneumonia. Arch. Pediatr. Adolesc. Med. 165, 506-512 (2011).

8. Hageman, J. C. et al. Severe community-acquired pneumonia due to Staphylococcus aureus, 2003-04 influenza season. Emerg. Infect. Dis. 12, 894-899 (2006).

9. Kallen, A. J. et al. Staphylococcus aureus community-acquired pneumonia during the 2006 to 2007 influenza season. Ann. Emerg. Med. 53, 358-365 (2009).

10. Lejeune, D. et al. Interleukin-22 (IL-22) activates the JAK/STAT, ERK, JNK, and p38 MAP kinase pathways in a rat hepatoma cell line. Pathways that are shared with and distinct from IL-10. J. Biol. Chem. 277, 33676-33682 (2002).

11. Liang, S. C. et al. Interleukin (IL)-22 and IL-17 are coexpressed by Th17 cells and cooperatively enhance expression of antimicrobial peptides. J. Exp. Med. 203, 2271-2279 (2006).

12. Wolk, K. et al. IL-22 increases the innate immunity of tissues. Immunity 21, 241-254 (2004)

13. Aujla, S. J. et al. IL-22 mediates mucosal host defense against Gram-negative bacterial pneumonia. Nat. Med. 14, 275-281 (2008).

14. Trevejo-Nunez, G., Elsegeiny, W., Conboy, P., Chen, K. \& Kolls, J. K. Critical role of IL-22/IL22-RA1 signaling in Pneumococcal pneumonia. J. Immunol. 197, 1877-1883 (2016).

15. Dumoutier, L., Lejeune, D., Colau, D. \& Renauld, J. C. Cloning and characterization of IL-22 binding protein, a natural antagonist of IL-10-related T cell-derived inducible factor/IL-22. J. Immunol. 166, 7090-7095 (2001).

16. Kotenko, S. V. et al. Identification, cloning, and characterization of a novel soluble receptor that binds IL-22 and neutralizes its activity. J. Immunol. 166, 7096-7103 (2001).

17. $\mathrm{Xu}, \mathrm{W}$. et al. A soluble class II cytokine receptor, IL-22RA2, is a naturally occurring IL-22 antagonist. Proc. Natl Acad. Sci. USA 98, 9511-9516 (2001).

18. Laaksonen, $\mathrm{H}$. et al. The multiple sclerosis risk gene IL22RA2 contributes to a more severe murine autoimmune neuroinflammation. Genes Immun. 15, 457-465 (2014).

19. Sertorio, M. et al. IL-22 and IL-22 binding protein (IL-22BP) regulate fibrosis and cirrhosis in hepatitis $\mathrm{C}$ virus and schistosome infections. Hepatology 61 1321-1331 (2015).

20. Ratsimandresy, R. A., Indramohan, M., Dorfleutner, A. \& Stehlik, C. The AIM2 inflammasome is a central regulator of intestinal homeostasis through the IL-18/ IL-22/STAT3 pathway. Cell. Mol. Immunol. 14, 127-142 (2017).

21. Broquet, A. et al. Interleukin-22 level is negatively correlated with neutrophil recruitment in the lungs in a Pseudomonas aeruginosa pneumonia model. Sci. Rep. 7, 11010 (2017).

22. Kudva, A. et al. Influenza A inhibits Th17-mediated host defense against bacterial pneumonia in mice. J. Immunol. 186, 1666-1674 (2011).

23. Barthelemy, A. et al. Interleukin-22 immunotherapy during severe influenza enhances lung tissue integrity and reduces secondary bacterial systemic invasion. Infect. Immun. 86, pii: e00706-17 (2018).

24. Ivanov, S. et al. Interleukin-22 reduces lung inflammation during influenza $A$ virus infection and protects against secondary bacterial infection. J. Virol. 87, 6911-6924 (2013).

25. Herold, S. et al. Lung epithelial apoptosis in influenza virus pneumonia: the role of macrophage-expressed TNF-related apoptosis-inducing ligand. J. Exp. Med. 205, 3065-3077 (2008).

26. Peteranderl, C. et al. Macrophage-epithelial paracrine crosstalk inhibits lung edema clearance during influenza infection. J. Clin. Invest. 126, 1566-1580 (2016).

27. Dawson, T. C., Beck, M. A., Kuziel, W. A., Henderson, F. \& Maeda, N. Contrasting effects of CCR5 and CCR2 deficiency in the pulmonary inflammatory response to influenza A virus. Am. J. Pathol. 156, 1951-1959 (2000).

28. Lin, K. L., Suzuki, Y., Nakano, H., Ramsburg, E. \& Gunn, M. D. CCR2+ monocytederived dendritic cells and exudate macrophages produce influenza-induced pulmonary immune pathology and mortality. J. Immunol. 180, 2562-2572 (2008).

29. Ghoneim, H. E., Thomas, P. G. \& McCullers, J. A. Depletion of alveolar macrophages during influenza infection facilitates bacterial superinfections. J. Immunol. 191, 1250-1259 (2013).
30. Kumar, P., Thakar, M. S., Ouyang, W. \& Malarkannan, S. IL-22 from conventional NK cells is epithelial regenerative and inflammation protective during influenza infection. Mucosal Immunol. 6, 69-82 (2013).

31. Paget, $C$. et al. Interleukin-22 is produced by invariant natural killer T lymphocytes during influenza A virus infection: potential role in protection against lung epithelial damages. J. Biol. Chem. 287, 8816-8829 (2012).

32. Pociask, D. A. et al. IL-22 is essential for lung epithelial repair following influenza infection. Am. J. Pathol. 182, 1286-1296 (2013).

33. Gunzel, D. \& Yu, A. S. Claudins and the modulation of tight junction permeability. Physiol. Rev. 93, 525-569 (2013).

34. Wittekindt, O. H. Tight junctions in pulmonary epithelia during lung inflammation. Pflug. Arch. 469, 135-147 (2017).

35. Robinson, K. M. et al. Influenza A virus exacerbates Staphylococcus aureus pneumonia in mice by attenuating antimicrobial peptide production. J. Infect. Dis. 209, 865-875 (2014).

36. Robinson, K. M. et al. The role of IL-27 in susceptibility to post-influenza Staphylococcus aureus pneumonia. Respir. Res. 16, 10 (2015).

37. Planet, P. J. et al. Lambda interferon restructures the nasal microbiome and increases susceptibility to Staphylococcus aureus superinfection. mBio 7, e01939-01915 (2016).

38. Huber, S., Gagliani, N., Zenewicz, L. A., Huber, F. J., Bosurgi, L. \& Hu, B. et al. IL$22 \mathrm{BP}$ is regulated by the inflammasome and modulates tumorigenesis in the intestine. Nature 491, 259-263 (2012).

39. Martin, J. C. et al. Interleukin-22 binding protein (IL-22BP) is constitutively expressed by a subset of conventional dendritic cells and is strongly induced by retinoic acid. Mucosal Immunol. 7, 101-113 (2014).

40. Kash, J. C. et al. Genomic analysis of increased host immune and cell death responses induced by 1918 influenza virus. Nature 443, 578-581 (2006).

41. Kobasa, D. et al. Aberrant innate immune response in lethal infection of macaques with the 1918 influenza virus. Nature 445, 319-323 (2007).

42. Pelczar, P. et al. A pathogenic role for T cell-derived IL-22BP in inflammatory bowel disease. Science 354, 358-362 (2016).

43. Sugimoto, K. et al. IL-22 ameliorates intestinal inflammation in a mouse model of ulcerative colitis. J. Clin. Invest. 118, 534-544 (2008).

44. Kleinschmidt, D. et al. A protective function of IL-22BP in ischemia reperfusion and acetaminophen-induced liver injury. J. Immunol. 199, 4078-4090 (2017).

45. Munoz, M. et al. Interleukin (IL)-23 mediates Toxoplasma gondii-induced immunopathology in the gut via matrixmetalloproteinase-2 and IL-22 but independent of IL-17. J. Exp. Med. 206, 3047-3059 (2009).

46. Martin, J. C. et al. Limited presence of IL-22 binding protein, a natural IL-22 inhibitor, strengthens psoriatic skin inflammation. J. Immunol. 198, 3671-3678 (2017).

47. Voglis, S. et al. Regulation of IL-22BP in psoriasis. Sci. Rep. 8, 5085 (2018).

48. Short, K. R. et al. Influenza virus damages the alveolar barrier by disrupting epithelial cell tight junctions. Eur. Respir. J. 47, 954-966 (2016).

49. Tian, T. et al. H3N2 influenza virus infection enhances oncostatin M expression in human nasal epithelium. Exp. Cell Res. 371, 322-329 (2018).

50. Ahn, D., Wickersham, M., Riquelme, S. \& Prince, A. The effects of IFN-lambda on epithelial barrier function contribute to Klebsiella pneumoniae ST258 pneumonia. Am. J. Respir. Cell Mol. Biol. 60, 158-166 (2019).

51. Wang, Y., Mumm, J. B., Herbst, R., Kolbeck, R. \& Wang, Y. IL-22 increases permeability of intestinal epithelial tight junctions by enhancing claudin-2 expression. J. Immunol. 199, 3316-3325 (2017).

52. Robinson, K. M., Kolls, J. K. \& Alcorn, J. F. The immunology of influenza virus associated bacterial pneumonia. Curr. Opin. Immunol. 34, 59-67 (2015).

53. Lim, C., Hong, M. \& Savan, R. Human IL-22 binding protein isoforms act as a rheostat for IL-22 signaling. Sci. Signal. 9, ra95 (2016).

54. Gopal, R. et al. STAT2 signaling regulates macrophage phenotype during influenza and bacterial super-infection. Front. Immunol. 9, 2151 (2018).

55. Lee, B. et al. STAT1 is required for suppression of type 17 immunity during influenza and bacterial superinfection. Immunohorizons 1, 81-91 (2017).

56. Van der Velden, J. et al. Differential requirement for c-Jun $\mathrm{N}$-terminal kinase 1 in lung inflammation and host defense. PLOS ONE 7, e34638 (2012).

57. Manni, M. L. et al. Bromodomain and extra-terminal protein inhibition attenuates neutrophil-dominant allergic airway disease. Sci. Rep. 7, 43139 (2017).

58. Cozens, A. L. et al. CFTR expression and chloride secretion in polarized immortal human bronchial epithelial cells. Am. J. Respir. Cell Mol. Biol. 10, 38-47 (1994).

59. Hendricks, M. R. et al. Respiratory syncytial virus infection enhances Pseudomonas aeruginosa biofilm growth through dysregulation of nutritional immunity. Proc. Natl Acad. Sci. USA 113, 1642-1647 (2016).

60. Bomberger, J. M. et al. Long-distance delivery of bacterial virulence factors by Pseudomonas aeruginosa outer membrane vesicles. PLoS Pathog. 5, e1000382 (2009). 